\title{
Vorwort zur „Technischen Entwicklung auf dem Gebiete der Kohlenwasserstofföle und Fette“.
}

I'naufhaltsam vollzieht sich der Fortschritt der Wissenschaft und der Technik. Die Erfolge beider dienen der Wirtschaft und diese wirkt wieder befruchtend auf die beiden Erstgenannten in glücklichem Wechsel.

Der Techniker aber, der in diesem Getriebe steht, sieht sich gleichsam einem stetig anschwellenden Strom von Tatsachen gegenüber, der ihm in seinen Fachzeitschriften zugetragen wird und den er neben den schweren Aufgaben des Tagesbetriehes in seiner Gesamtheit kaum mehr zu meistern imstande ist. Scit dem Kriege, der unter anderen İbeln auch die für Deutschland gewiß n'cht erwünschte Belebung der chemischen Industrie des Auslandes gezeitigt hat, ist dieser Tatsachenstrom noch stärker geworden und damit die Not des Technikers, mit der Entwicklung des Faches Schritt zu halten.

Das vorliegende Werk w'll nun diesen Schwierigkeiten entgeger1treten, indem $\mathrm{es}$ den Tatsachenstrom in einzelne gut ausgebaute Kanäle lcitet oder, ohne Bild gesprochen, das Material ordnet und sichtet e t w a in der Art, wie das auf dem Farbstoffgebicte durch F riedlä nde r s Fortschritte der Farbenfabrikation und, auf dem Gebiete der anorganischen Chemie, durch das Werk von Brä u er-d'Ans geschehen ist. -

Hier handelt es cich also um die Forts chritte in der Te chnologie der Kohlenwasserstofföle und Fette.

Das Werk hat die Aufgabe, die internationale Patentliteratur dieser Zweige, im wesentlichen seit 1921, zu sammeln.

Während bei den eben erwähnten Werken nur die deutschen Patente Berücksichtigung fanden, ergab eine İberprüfung unseres Gebieteৎ, daß das hier nicht genügte, daß man vieimehr daran gehen müßte, einen möglichst großen Ausschnitt aus dem ausländischen Patentschrifttum heranzuziehen, wenn man das Ziel, das uns vorschwebte, erreichen wollte, das Ziel, de $\mathrm{n} \mathrm{F} \mathrm{a} \mathrm{ch}$ gen os sen ein m ögli ch st 
genaues Bild der technischen Entwicklung zu g e be n und zwar vom Standpunkte des technischen Chemikers.

Die Patentliteratur des Auslandes wurde unmittelbar bearbeitet, soweit es sich um die amerikanischen, englischen, französischen, kanadischen, belgischen, österreichischen und schweizer Patente handelt, die sonstigen, uns sprachlich nicht zugänglichen Patentschriften, soweit sie das Chem. Zentralblatt vermittelt.

Als technische Umgrenzung haben wir die Stoffe vom Rohmaterial an berücksichtigt, daß heißt unter Ausschluß der Verfahren zur Gewinnung von Erdöl und Kohle oder allg eme in er Verfahren zur Destillation und Raffination, die in Spezialwerken zu finden sind.

Die Einteilung und Verarbeitung der Stoffe erfolgt nach Möglichkeit in der Weise, daß jeder Band für sich eine vollständige Wiedergabe des einschlägigen Gebietes enthält, ohne daß in erheblichem Maße Hinweise auf andere Bände nötig werden. Jeder Band stellt also ein selbständiges Werk und eine selbständige Zusammenfassung der technischen Entwicklung auf seinem Gebiete in den letzten 5 Jahren dar. Doch werden auch Patente aus früheren Jahren berücksichtigt, wenn das zur Abrundung der Darstellung erforderlich scheint. 